\title{
Atherosclerotic Plaques Recognition in Intracoronary Optical Images Using Neural Networks
}

\author{
Maysa Malfiza Garcia de Macedo ${ }^{1}$, Dario Augusto Borges Oliveira ${ }^{1}$, Marco Antonio Gutierrez ${ }^{2}$ \\ ${ }^{1}$ IBM Research, São Paulo, Brazil \\ ${ }^{2}$ Heart Institute (InCor), University of São Paulo Medical School, São Paulo, Brazil
}

\begin{abstract}
Coronary artery disease $(C A D)$ is intrinsically related to presence of atherosclerotic plaques. The rupture of this plaques is responsible for most acute coronary events. Intracoronary optical coherence tomography (IOCT) enables a detailed high-resolution visualization of microstructural changes of the arterial wall in vivo. In this paper, we introduce a new way of identifying atherosclerotic plaques using 1D Convolutional Neural Networks (CNN) analyzing only the lumen contour. Training and test were performed with 1600 IOCT frames from in vivo patients. In our tests, we achieved fl-score of $95 \%$ for atherosclerotic plaque recognition. The results allow us to report an interesting correlation between the lumen contour geometry and the presence of plaques in the vascular wall observed through IOCT exams. The use of lumen contour for plaque detection opens two new perspectives: assisting specialists in the task of detecting plaques visually by paying special attention to the lumen and allowing methods to work in real time to detect plaques using efficient methods that use less information and deliver accurate results.
\end{abstract}

\section{Introduction}

Coronary atherosclerotic plaques are inflamed regions in the coronary wall that may be mainly composed of three materials: collagen, lipid and calcium. These plaques are one of the consequences of coronary artery disease (CAD) that today is responsible for high mortality taxes worldwide [1]. Intravascular Optical Coherence Tomography (IOCT) imaging have been used clinically in cardiac catheterizing exams since 2010 and provides high resolution images of the vascular wall. Yabushita et al [2] attested IOCT can accurately characterize plaque components with a high degree of sensitivity and specificity. While it is possible to visualize and quantify plaques in these exams, the amount of images to be analyzed for each exam make the task of quantifying the plaques extremely exhaustive and time-consuming.
Several papers address atherosclerotic plaques classification in IOCT through the analysis of $2 \mathrm{D}$ slices of the vascular wall and propose methods usually very related to texture pattern recognition $[3,4]$. For instance, Athanasiou et al. [5] used Random Forest to analyze textures and classify different plaques and [6] proposed a method to detect and classify plaques between calcified and fibrous/lipid using deep learning model, such as Resnet50 and Densenet121. Macedo et al. [7] proposed to identify side branches in slices using only the lumen contour and reported that irregular geometry in slices with plaque was the main cause of confusion since regular slices without plaques present a nearly circular lumen shape.

In this work we explore the premise that usually atherosclerotic plaques cause deformations in the lumen and propose a method for identifying plaques using only the lumen geometry. We use a 1D-convolutional neural network that takes as input the lumen outline by means of distances to the lumen centroid and perform a plaque detection using this information. Our hypothesis is that plaques are detectable using simply lumen contour information, manually or automatically delineated. To the best of our knowledge, this is the first paper to use only contour for plaque detection (Boi et al [8]).

\section{Method}

\subsection{Data}

The data used is composed of IOCT images acquired using a Fourier-Domain IOCT system from Heart Institute - University of São Paulo Medical School - Brazil with the approved study protocol CAPPesq under register n. 0243/08. In total, 1600 2D images from 9 patients were used to detect side-branches and from this total 1389 2D images were used to detect plaques (discarding sidebranch samples). We used 5 patients (914 2D images) for training, 2 patients (356 2D images) for validation and 2 patients ( 330 2D images) for testing, distributed as depicted in Table 1. The 2D input images were acquired in DICOM format and the dimensions of $704 \times 704$ pixels, 

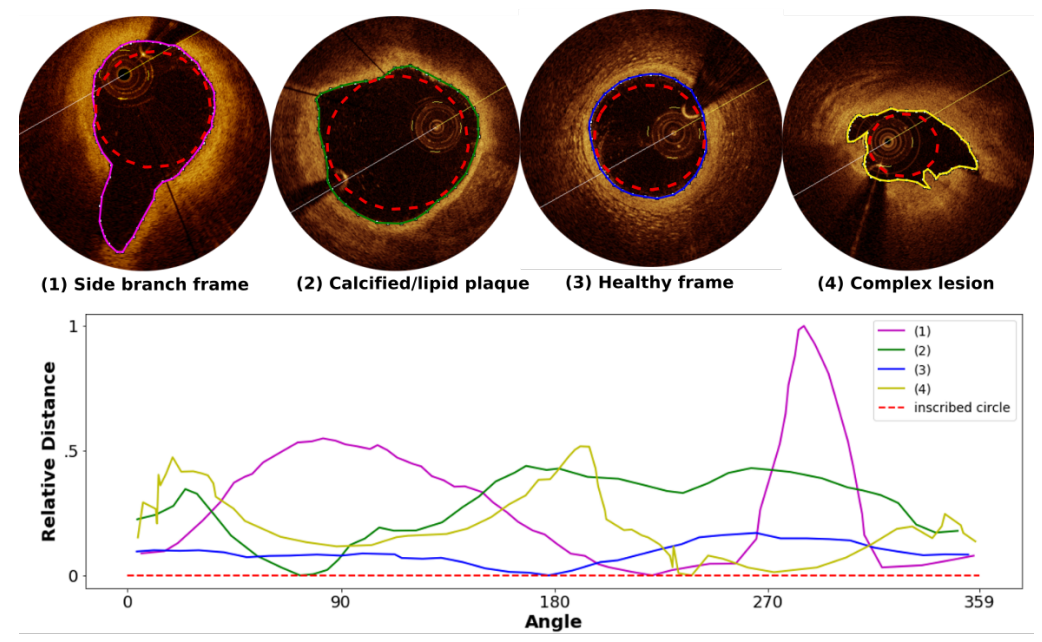

Figure 1. Example of IOCT frame samples with the lumen contour used in our experiments. The graphic below depicts the relative distance between centroid lumen and the lumen contour points.

with axial resolution of $10 \mu \mathrm{m}$ and lateral resolution of 20-40 $\mu \mathrm{m}$. Pullbacks speed was set as $20 \mathrm{~mm} / \mathrm{sec}$ over a distance of $54.0 \mathrm{~mm}$.

Table 1. Number of samples for training, testing and validation datasets.

\begin{tabular}{c|c|c|c|c}
\hline \hline Dataset & not-plaque & plaque & side-branch & \#total \\
\hline training & 63 & 729 & 122 & 914 \\
validation & 54 & 257 & 45 & 356 \\
testing & 49 & 237 & 44 & 330 \\
\hline \hline
\end{tabular}

All 1600 frames were visually inspected and annotated by an expert, which was asked to: 1-identify if there is plaque or not in the frame; 2- identify if the frame is in side-branch; and 3- delineate the lumen contour. Although we used manually delineated contours in this paper, many successful methods for automated contour delineation are available in the literature, such as $[9,10]$, with impressive results in comparison to manual counterparts and could be used as input. Figure 1 shows some examples of IOCT frames with the annotated lumen contour.

\subsection{Pre-processing}

To create samples for training our network, we defined an array of distances for each available lumen contour. For that, we considered a relative distance vector to an imaginary inscribed circle, in such a way that a perfectly circular lumen would derive a zero-valued array. Irregularities in the lumen contour are modelled as distances to this imaginary circle, creating a vector of distances that is used as input data for the proposed 1D convolutional neural network, as depicted in Figure 1. One can observe that a healthy frame, such the one depicted in (3), has a nearly circular contour, and therefore, low distance values, while different anomalies present different patterns in the contour and consequently in the distances array.

For dealing with the unbalanced nature of our database - presented in table 1, we performed a simple data augmentation of healthy vascular walls that consists of rolling sequentially with a shift of 10 positions, augmenting 10 times the number of samples for this class. This process derived a training database for plaques detection with roughly 630 samples for healthy walls and 729 for walls with plaques. For the side branch detection, we did augmentation for both classes, deriving 630 samples for regular walls, and 610 for side branching using shift of 20 positions instead of 10 . Validation and testing sets were not augmented in any experiment.

\subsection{Model}

We propose to use one-dimensional CNN architecture, as depicted in Figure 2. This simple one-dimensional CNN architecture was successfully used for 1D analysis in [11] and receives a 1D array as input to be processed by a sequence of 1D convolutional layers with ReLu activation and processed by a dense layer block that delivers a final outcome. In this model, each one-dimensional convolutional layer is followed by a MaxPooling layer with a kernel size of 2 pixels.

The model used in our experiments has three hyperparameters, as depicted in Figure 2: the number of convolutional blocks, number of filters in each block, and the number of nodes in the dense layer. We experimentally tuned them considering a range of 1 to 5 convolutional blocks, number of filters in each block assuming all pos- 


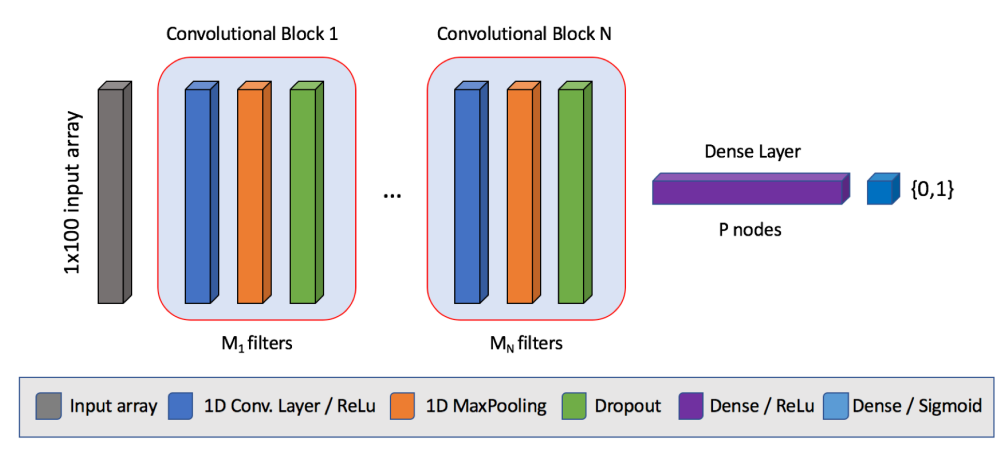

Figure 2. Our 1-D CNN model architecture: a convolutional block composed by a number of convolutional layers, followed by a dense block for binary classification.

sible values in $\{32,64,128,256,512\}$, and number of dense layer nodes ranging from 10 to 150 . We report the best configurations in Table 2. For training we used Adam optimizer with learning rate of 0.001 , bet $a_{1}=0.9$ and bet $a_{2}=0.999$. We trained our models until convergence, adopting the early stop criteria considering validation accuracy drop.

\section{Results}

To validate our methodology, we propose two experiments: one for plaque detection and the other for sidebranch detection - which allow us to compare our method with the literature and increase trust in our model for plaque detection.

We varied the number of layers and filters per layer to find the optimal model for each experiment. For side branch detection we observed the same results for a wide range of hyper-parameters, while in plaques detection the best models were associated to a larger number of convolutional layers and larger number of filters. This is probably due to the subtler pattern observed in lumen geometry with presence of plaques, in comparison to more drastic variations for side branching.

Our results for side branch detection achieved 97\% of area under the curve (AUC), while plaque detection achieved AUC of $83 \%$. Several commonly used evaluation metrics for binary classification are shown in Table 2, considering different model configurations that ranked among the best results for detection of side branches and atherosclerotic plaques. The corresponding ROC curves are presented in Figure 3.

\section{Discussion}

During our experiments we could clearly observe that side-branch detection present better results in comparison to plaques detection using the lumen geometry, as ex-
Table 2. Results for side-branch detection and plaque detection using lumen geometry. Models are represented as the sequence of convolutional blocks in brackets with the corresponding number of filters in each, followed by the number of nodes in the dense layer.

\begin{tabular}{c|c|c|c|c|c}
\hline Experiment & Se & Sp & Acc & f1-sc & AUC \\
\hline \hline \multicolumn{7}{c}{ Side branch detection } \\
\hline$[128]-150$ & 0.51 & $\mathbf{0 . 9 9}$ & 0.87 & 0.92 & $\mathbf{0 . 9 7}$ \\
{$[64,128]-100$} & $\mathbf{0 . 7 3}$ & 0.98 & $\mathbf{0 . 9 4}$ & $\mathbf{0 . 9 6}$ & 0.96 \\
{$[32,64]-100$} & 0.5 & $\mathbf{0 . 9 9}$ & 0.87 & 0.92 & $\mathbf{0 . 9 7}$ \\
{$[64,128,256]-150$} & 0.65 & $\mathbf{0 . 9 9}$ & 0.92 & 0.95 & $\mathbf{0 . 9 7}$ \\
\hline \multicolumn{7}{c}{ Plaque detection } \\
\hline$[256,512]-100$ & 0.67 & 0.94 & 0.89 & 0.93 & $\mathbf{0 . 8 3}$ \\
{$[64,128]-30$} & 0.72 & 0.94 & 0.91 & 0.94 & 0.82 \\
{$[128,256,512]-100$} & 0.66 & $\mathbf{0 . 9 5}$ & 0.89 & 0.93 & 0.81 \\
{$[32,64,128]-100$} & $\mathbf{0 . 7 3}$ & $\mathbf{0 . 9 5}$ & $\mathbf{0 . 9 1}$ & $\mathbf{0 . 9 5}$ & $\mathbf{0 . 8 3}$ \\
\hline
\end{tabular}

pected. Plaques can be small or with a density that promotes slight deformations of the lumen, or can also deform the lumen in varied forms, while bifurcations seem to have a clearer pattern in the changes of lumen geometry. While many different configurations achieved satisfactory performances for side-branch detection, optimization of hyper-parameters seem to be important for achieving good performances for plaques detection.

We also compared our side-branch detection outcome with the method proposed in Macedo et al. [7] since they share great part of the database. For side-branch detection, we report an increase of $2.4 \%$ of AUC for such task (97\% against $94.61 \%$ in [7]), and this finding encourages us to hypothesize that the proposed 1-D CNN model can also provide plausible results for plaque detection - considering that lumen geometry is a discriminant feature for such task.

When it comes to plaques detection in IOCT, the survey of Boi et al. [8] describes that methods using texture information for plaque detection and classification performs 


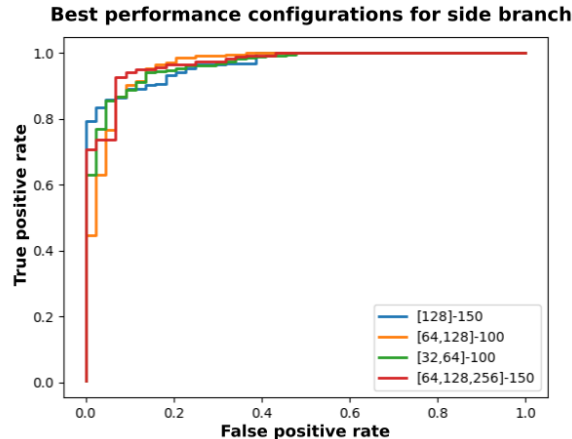

(a)

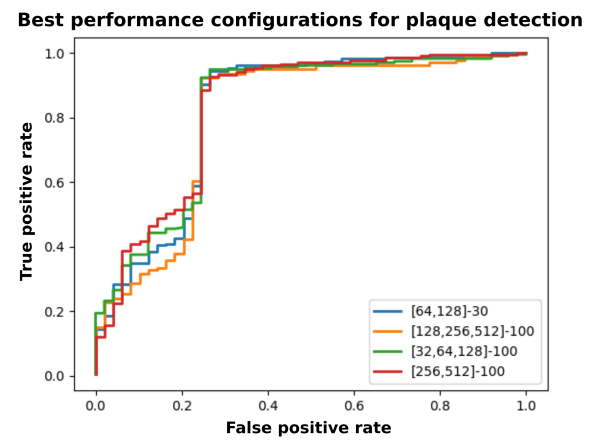

(b)

Figure 3. Receiver operating characteristic (ROC) curve for (a) side-branch detection and (b) plaque detection. Models are represented as the sequence of convolutional blocks followed by the number of nodes in the dense layer.

with accuracy ranging between $80.41 \%$ and $88 \%$. The recent paper of Gessert et al. [6] report an accuracy of $92 \%$ for plaque detection using texture information. It is interesting to notice that our method, that takes into account only the lumen geometry was able to achieve $91 \%$ of accuracy.

\section{Conclusion}

This paper presents a method based on 1D convolutional neural networks that uses lumen geometry to detect plaques in IOCT images. Our results allow us to report an interesting correlation between the lumen contour geometry and the presence of plaques in the vascular wall observed through IOCT exams. The use of lumen contour for plaque detection opens two new perspectives: assisting specialists in the task of detecting plaques visually by paying special attention to the lumen and allowing automated methods to work in real time to detect plaques using efficient methods that use less information and deliver accurate results. Further research includes classifying types of plaques considering the lumen geometry and understanding how different tissues contributes to deformations observed in the lumen.

\section{References}

[1] WHO Cardiovascular disease. who.int/mediacentre/factsheets/fs317.

[2] Yabushita H. Characterization of human atherosclerosis by optical coherence tomography. Circulation sep 2002; 106(13):1640-1645. ISSN 00097322.

[3] Zahnd G, Karanasos A. Quantification of fibrous cap thickness in intracoronary optical coherence tomography with a contour segmentation method based on dynamic programming. International Journal of Computer Assisted Radiology and Surgery 2015;1383-1394. ISSN 1861-6410.

[4] Ughi GJ, Adriaenssens T, Sinnaeve P, Desmet W, D'hooge J. Automated tissue characterization of in vivo atherosclerotic plaques by intravascular optical coherence tomography images. Biomedical Optics Express jul 2013; 4(7):1014-30. ISSN 2156-7085.

[5] Athanasiou LS, Bourantas CV, Rigas G, Sakellarios AI, Exarchos TP, Siogkas PK, et al. Methodology for fully automated segmentation and plaque characterization in intracoronary optical coherence tomography images. Journal of Biomedical Optics feb 2014;19(2):026009. ISSN 10833668.

[6] Gessert N, Lutz M, Heyder M, Leistner SLDM, Abdelwahed YS, Schlaefer A. Automatic plaque detection in IVOCT pullbacks using convolutional neural networks. IEEE Transactions on Medical Imaging 2018;(August). ISSN 0278-0062.

[7] Macedo MMG, Guimarães WVN, Galon MZ, Takimura CK, Lemos PA, Gutierrez MA. A bifurcation identifier for IV-OCT using orthogonal least squares and supervised machine learning. Computerized medical imaging and graphics sep 2015;46(2):237-248. ISSN 1879-0771.

[8] Boi A, Jamthikar AD, Saba L, Gupta D, Sharma A, Loi B, Laird JR, Khanna NN, Suri JS. A survey on coronary atherosclerotic plaque tissue characterization in intravascular optical coherence tomography 2018;.

[9] de Macedo M, Takimura C, Lemos P, Gutierrez M. A robust fully automatic lumen segmentation method for in vivo intracoronary optical coherence tomography. Revista Brasileira de Engenharia Biomedica 2016;32(1). ISSN 19847742 .

[10] Tsantis S, Kagadis GC, Katsanos K, Karnabatidis D, Bourantas G, Nikiforidis GC. Automatic vessel lumen segmentation and stent strut detection in intravascular optical coherence tomography. Medical Physics jan 2012; 39(1):503-13. ISSN 0094-2405.

[11] Oliveira DAB, Viana MP. Fast cnn-based document layout analysis. In 2017 IEEE International Conference on Computer Vision Workshops (ICCVW). ISSN 2473-9944, Oct 2017; 1173-1180.

Address for correspondence:

Maysa M G Macedo

Rua Tutoia, 1157, Sao Paulo, Brazil, 04007-900

\{mmacedo,dariobo\}@br.ibm.com 